Author affiliations appear at the end of this article.

Published online ahead of print at www.jco.org on July 15, 2013.

Written on behalf of the European T-Cell Lymphoma Study Group and International Peripheral T-Cell Lymphoma Project.

Supported by Centro Interdipartimentale per la Ricerca sul Cancro "G. Prodi," BolognAIL, Associazione Italiana per la Ricerca sul Cancro, Fondazione Cassa di Risparmio in Bologna, Fondazione della Banca del Monte e Ravenna, and Progetto Strategico di Ateneo.

Authors' disclosures of potential conflicts of interest and author contributions are found at the end of this article.

This study was investigator initiated and represents a major collaborative effort among involved hospitals. Funding bodies had no role in data collection, analysis, or interpretation; writing of the report; or decision to submit for publication. P.P.P. and S.A.P. had complete access to data and full responsibility for decision to submit the report.

Bologna University obtained a patent from the Italian Patent and Trademark Office for the molecular signatures used for the diagnostic classifier (Patent No. 61.U2164.12.IT.34).

Corresponding author: Pier Paolo Piccaluga, MD, PhD, Molecular Pathology Laboratory, Hematopathology Section, Department of Experimental, Diagnostic, and Specialty Medicine, S. Orsola-Malpighi Hospital, University of Bologna, Via Massarenti, 9 - 40138 Bologna, Italy; e-mail: pierpaolo piccaluga@unibo.it.

(C) 2013 by American Society of Clinical Oncology

0732-183X/13/3124w-3019w/\$20.00

DOI: $10.1200 / J C O .2012 .42 .5611$

\title{
Molecular Profiling Improves Classification and Prognostication of Nodal Peripheral T-Cell Lymphomas: Results of a Phase III Diagnostic Accuracy Study
}

Pier Paolo Piccaluga, Fabio Fuligni, Antonio De Leo, Clara Bertuzzi, Maura Rossi, Francesco Bacci, Elena Sabattini, Claudio Agostinelli, Anna Gazzola, Maria Antonella Laginestra, Claudia Mannu, Maria Rosaria Sapienza, Sylvia Hartmann, Martin L. Hansmann, Roberto Piva, Javeed Iqbal, John C. Chan, Denis Weisenburger, Julie M. Vose, Monica Bellei, Massimo Federico, Giorgio Inghirami, Pier Luigi Zinzani, and Stefano A. Pileri

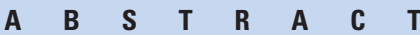

\section{Purpose}

The differential diagnosis among the commonest peripheral T-cell lymphomas (PTCLs; ie, PTCL not otherwise specified [NOS], angioimmunoblastic T-cell lymphoma [AITL], and anaplastic large-cell lymphoma [ALCL]) is difficult, with the morphologic and phenotypic features largely overlapping. We performed a phase III diagnostic accuracy study to test the ability of gene expression profiles (GEPs; index test) to identify PTCL subtype.

\section{Methods}

We studied 244 PTCLs, including 158 PTCLs NOS, 63 AITLs, and 23 ALK-negative ALCLs. The GEP-based classification method was established on a support vector machine algorithm, and the reference standard was an expert pathologic diagnosis according to WHO classification.

\section{Results}

First, we identified molecular signatures (molecular classifier [MC]) discriminating either AITL and ALK-negative ALCL from PTCL NOS in a training set. Of note, the MC was developed in formalin-fixed paraffin-embedded (FFPE) samples and validated in both FFPE and frozen tissues. Second, we found that the overall accuracy of the MC was remarkable: $98 \%$ to $77 \%$ for AITL and $98 \%$ to $93 \%$ for ALK-negative ALCL in test and validation sets of patient cases, respectively. Furthermore, we found that the MC significantly improved the prognostic stratification of patients with PTCL. Particularly, it enhanced the distinction of ALK-negative ALCL from PTCL NOS, especially from some CD30+ PTCL NOS with uncertain morphology. Finally, MC discriminated some T-follicular helper (Tfh) PTCL NOS from AITL, providing further evidence that a group of PTCLs NOS shares a Tfh derivation with but is distinct from AITL.

\section{Conclusion}

Our findings support the usage of an MC as additional tool in the diagnostic workup of nodal PTCL.

\section{J Clin Oncol 31:3019-3025. (C) 2013 by American Society of Clinical Oncology}

\section{INTRODUCTION}

Peripheral T-cell lymphomas (PTCLs) represent $10 \%$ to $15 \%$ of all lymphomas, and their incidence is higher in Asia than in Western countries. ${ }^{1}$ They include several different entities ${ }^{1}$; however, four main subtypes (ie, PTCL not otherwise specified [NOS], angioimmunoblastic T-cell lymphoma [AITL], and anaplastic large-cell lymphoma [ALCL; ALK negative and ALK positive]) represent approximately $75 \%$ of all patient cases in Europe and the United States. ${ }^{1}$ The diagnosis of PTCL is usually challenging, requiring expertise in hematopathology. In this regard, a large international study recently reported that up to $30 \%$ of PTCLs are misdiagnosed. ${ }^{2}$ Addi- tionally, the differential diagnosis among nodal PTCLs may be affected by subjective criteria. In fact, the morphology and phenotype of PTCL NOS is quite variable, the former including features usually observed in AITL or ALCL (ie, clear follicular Thelper-related or large CD30+ cells, respectively). Moreover, only a fraction of ALCLs carry a specific genetic lesion- $\mathrm{t}(2 ; 5)$ and variants, all leading to ALK aberrant expression-defining a distinct entity in WHO classification. However, the distinction of PTCL NOS from either AITL or ALK-negative ALCL is not trivial. In fact, ALK-negative ALCL and PTCL NOS are characterized by a different prognosis, ${ }^{3}$ and distinct therapeutic approaches have been proposed. ${ }^{4}$ 
Recently, gene expression profiling (GEP) was shown to be useful to discriminate different entities when morphology and phenotype are not consistent. ${ }^{5,6}$ In this regard, some reports have described the ability of GEP in differentiating PTCL NOS from ALCL or AITL. ${ }^{7-11}$ However, these studies used RNA from fresh/frozen samples and were not designed to test the diagnostic value of molecular signatures.

In this phase III study, we aimed first to test the diagnostic accuracy of GEP-based molecular classifiers (MCs) in the differential diagnosis between PTCL NOS and either AITL or ALK-negative ALCL, starting from formalin-fixed paraffin-embedded (FFPE) tissues, and second to evaluate the potential clinical implications.

\section{METHODS}

\section{Patient Case Selection}

We studied 244 nodal PTCLs, starting from either FFPE $(n=112)$ or fresh/frozen $(n=132)$ tissues. We included samples of PTCL NOS $(n=80)$, $\operatorname{AITL}(\mathrm{n}=20)$, and ALK-negative ALCL $(\mathrm{n}=12)$ for which FFPE blocks were available, and patients had given permission for analysis.

In addition, we studied 132 PTCLs (78 PTCLs NOS, 43 AITLs, and 11 ALK-negative ALCLs) as a validation set for which GEP had been previously generated from fresh/frozen biopsies by our group ${ }^{7-10}$ (Gene Expression Omnibus data sets GSE6338 and GSE19069). The latter did not correspond to any of the FFPE samples, having been recruited in a previous national study. ${ }^{7}$ Additional details are provided in the Data Supplement.

\section{GEP Generation and Analysis}

After RNA extraction, cDNA was prepared per manufacturer instructions, and gene expression analysis was carried out as previously reported, ${ }^{7,12,13}$ according to MIAME (Minimum Information About a Microarray Experiment) guidelines. Raw gene expression data will be available online after publication ${ }^{14}$ (details provided in Data Supplement).

\section{Diagnostic Accuracy Evaluation}

We tested the ability of two molecular signatures to discriminate either AITL or ALK-negative ALCL from PTCL NOS, respectively. Calculation of sensitivity (ST), specificity (SP), positive predictive value (PPV), negative predictive value (NPV), positive and negative likelihood ratio (LR), and odd ratio was performed using CATmaker software (Centre for Evidence-Based Medicine, Oxford University, Oxford, United Kingdom; http://www.cebm .net). The study was designed and conducted according to the STARD (Standards for the Reporting of Diagnostic Accuracy Studies) statement ${ }^{15}$ and REMARK (Reporting Recommendations for Tumor Marker Prognostic Studies) guidelines ${ }^{16}$ and informed by the QUADAS (Quality Assessment of Diagnostic Accuracy Studies) model. ${ }^{17}$ In particular, all patient cases were evaluated with both index (GEP-based classifier) and reference tests (histopathology, including complete immunohistochemistry, according to WHO classification), ${ }^{18}$ the two methods being completely independent.

\section{Survival Analyses}

Clinical information and complete follow-up were available for 203 of 244 patient cases. Specifically, overall survival (OS) was calculated from time of diagnosis to death or last follow-up. Statistical analyses were carried out using IBM SPSS Statistics 20.0 (IBM, Armonk, NY). Survival data were analyzed using the Kaplan-Meier and log-rank Mantle-Cox methods. ${ }^{19}$ The limit of significance for all analyses was defined as $P<.05$.

\section{RESULTS}

\section{AITL and ALK-Negative ALCL Can Be Distinguished From PTCL NOS Based on Global GEP}

Because unsupervised algorithms failed to clearly distinguish PTCL subtype (Data Supplement), we performed a supervised com- parison of PTCL NOS and either AITL or ALCL ( $t$ test with Bonferroni post hoc correction and Mann-Whitney test with BenjaminiHochberg post hoc correction, respectively) using a training set of FFPE samples (25 PTCLs NOS, 10 AITLs, and six ALK-negative ALCLs). Indeed, basing on $P$ value $(P<.05)$ and fold change $(\geq 2)$, we identified genes differentially expressed. In particular, we found 208 genes distinguishing PTCL NOS from AITL and 1,133 distinguishing PTCL/NOS from ALK-negative ALCL (Figs 1A and 1B; Data Supplement).

Subsequently, we performed a pathway analysis to assess whether the identified signatures corresponded to specific biologic functions. Remarkably, we found that genes distinguishing the different PTCLs were involved in relevant cellular programs. Specifically, genes discriminating PTCL/NOS from AITL were particularly involved in lipid metabolism, DNA replication, and regulation of cell cycle (Data Supplement). On the other hand, genes differentially expressed in PTCL/ NOS versus ALK-negative ALCL were significantly involved in regulation of apoptosis, protein kinase cascade, and immune response (Data Supplement).

Afterward, we investigated whether the identified molecular signatures could correctly distinguish AITL and ALK-negative ALCL samples from PTCL NOS by studying an independent test set of FFPE samples (55 PTCLs NOS, 10 AITLs, and six ALK-negative ALCL patient cases). Of note, by applying a hierarchic clustering, the different diseases were definitely discriminated (AITL $v$ PTCL NOS, $\chi^{2} P<$ .001 ; ALK-negative ALCL $v$ PTCL NOS, $\chi^{2} P<.001$; Figs 1C and 1D). Analog results were obtained in the validation set of fresh/frozen samples (Data Supplement).

\section{GEP-Based MC Is Efficient in Discriminating AITL and ALK-Negative ALCL From PTCL NOS}

We then focused on the possibility of developing a practical tool that could be applied in the routine diagnostic workup for the differential diagnosis of nodal PTCLs. To address this issue, we used a support vector machine algorithm, an easily reproducible system for GEP data management. First, we built the model by using all signatures in the training set of patient cases (25 PTCLs NOS, 10 AITLs, and six ALK-negative ALCLs) and then challenged it in the test set of patient cases (55 PTCLs NOS, 10 AITLs, and six ALK-negative ALCLs). Indeed, by applying the AITL prediction model, it was possible to correctly classify nine of 10 AITLs and 55 of 55 PTCLs NOS, with ST and SP of $90 \%$ and $100 \%$, respectively. Accordingly, PPV and NPV were calculated as $100 \%$ and $98 \%$, respectively, for an overall accuracy of $98 \%$ (Table 1; Data Supplement).

Alternatively, when we applied the ALK-negative ALCL prediction model, six of six ALCLs and 54 of 55 PTCLs NOS were correctly classified, with ST and SP of $100 \%$ and $98 \%$, respectively. PPV and NPV were then calculated as $86 \%$ and $100 \%$, respectively, for an overall accuracy of $98 \%$ (Table 2; Data Supplement).

We then tested the ability of MCs based on a more limited number of genes. Of note, by discriminant analysis, we could restrict the number of genes of the MC to 38 and 53 for AITL and ALKnegative ALCL, respectively, while maintaining the same accuracy.

Because these results seemed to have potential clinical relevance, to make our data more robust, we further tested the value of the assay in an independent data set (validation set) consisting of 78 PTCLs NOS, 43 AITLs and 11 ALK-negative ALCLs. Of note, these patient cases were originally studied starting from fresh/frozen material with a 


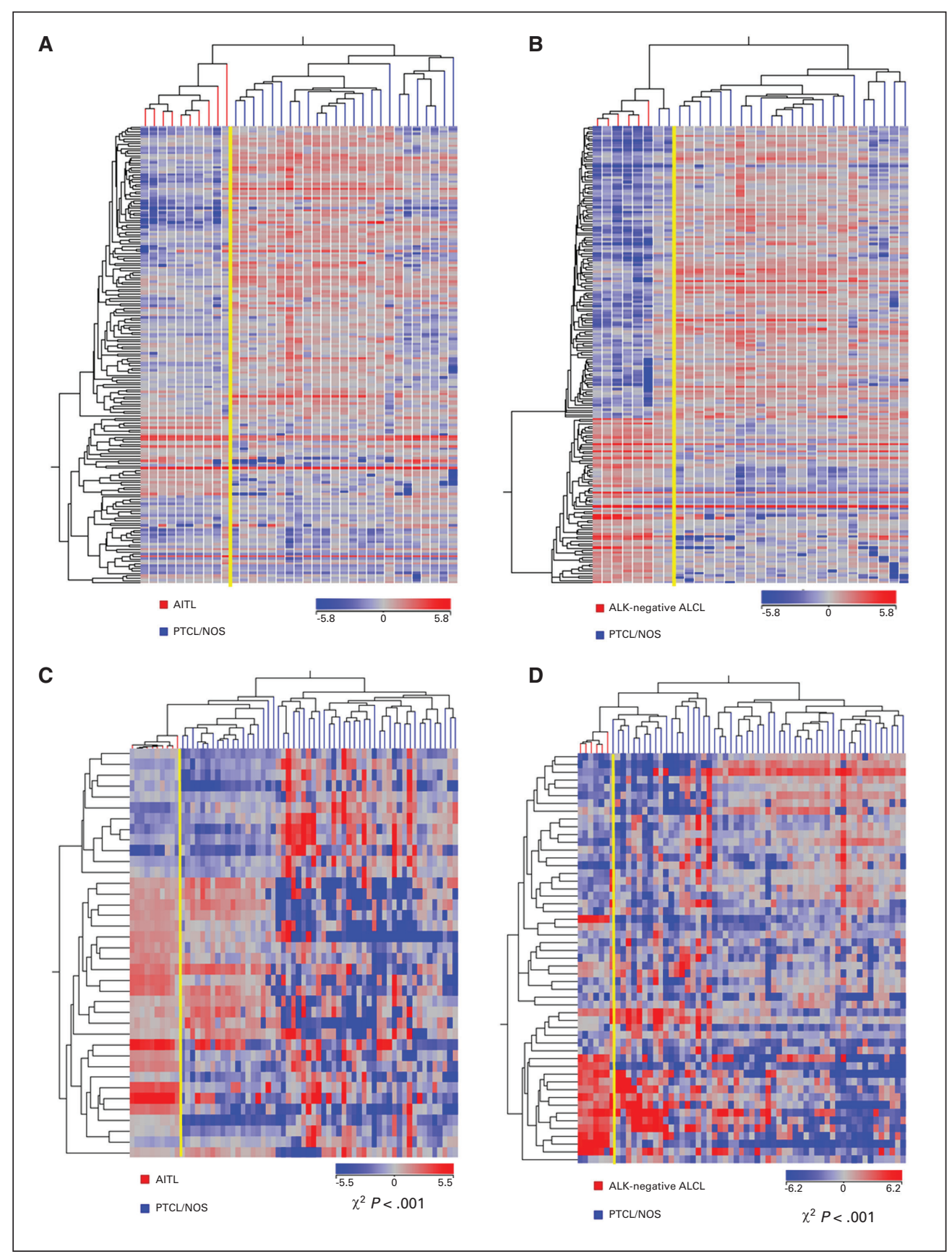

Fig 1. Supervised analyses identified genes differentially expressed in (A) angioimmunoblastic T-cell lymphoma (AITL) versus peripheral T-cell lymphoma (PTCL) not otherwise specified (NOS) and (B) ALK-negative anaplastic large-cell lymphoma (ALCL) versus PTCL (two-tailed $t$ test $P<.05$; fold change $\geq 2$ ). Top 200 genes in ranking are plotted. Unsupervised hierarchic clustering of nodal PTCLs constituting test set of patient cases based on genes differentially expressed in training set of (C) AITL versus PTCL NOS and (D) ALK-negative ALCL versus PTCL NOS (two-tailed $t$ test $P<.05$; fold change $\geq 2$ ). Top genes emerging from stepwise discriminant analysis are plotted. In matrix, each column represents sample, each row represents gene. Color scale bar shows relative gene expression changes normalized by standard deviation ( 0 is mean expression level of given gene). Clustering clearly distinguished AITL $\left(\chi^{2}\right.$ $P<.001)$ and $A L K$-negative $A L C L$ from PTCL NOS $\left(\chi^{2} P<.001\right)$.

different microarray; therefore, our analysis also served as additional technical control on the efficiency of the DASL (cDNA-Mediated Annealing, Selection, Extension, and Ligation) assay. In this case, with the AITL prediction model, 31 of 43 AITLs and 62 of 78 PTCLs NOS were correctly classified, with ST and SP of $72 \%$ and $80 \%$, respectively. Accordingly, PPV and NPV were $66 \%$ and $84 \%$, respectively, for an overall diagnostic accuracy of 77\% (Table 3; Data Supplement). Similarly, when PTCL NOS was tested against ALK-negative ALCL, eight of 11 ALCLs and 75 of 78 PTCLs NOS were correctly classified, with ST and SP of 73\% and 96\%, respectively. Therefore, PPV and NPV were then calculated as $73 \%$ and $96 \%$, respectively, for an overall diagnostic accuracy of 93\% (Table 4; Data Supplement). Together, these results indicate a remarkable diagnostic accuracy for the GEPbased MC in discriminating AITL and ALK-negative ALCL from PTCL NOS.

\section{MCs Significantly Affect Post-Test Probability of Disease}

Because a major factor in the indication of medical tests is their ability to make a difference between pre- and post-test probabilities of a given condition, we tested our MCs in this sense. Remarkably, concerning the discrimination of AITL from PTCL NOS, the post-test probability was increased from $15 \%$ to $100 \%$ and reduced from $15 \%$ to $2 \%$ for positive and negative results, respectively, in the test set. 


\begin{tabular}{|lcc|}
\hline \multicolumn{3}{|c}{ Table 1. Diagnostic Accuracy of AlTL Molecular Classifier Evaluated } \\
in Test Set
\end{tabular}

Similarly, it changed from $35 \%$ to $66 \%$ and to $16 \%$ for positive and negative results, respectively, in the validation set. Regarding the identification of ALK-negative ALCL, post-test probability was increased from $10 \%$ to $86 \%$ and reduced from $10 \%$ to $1 \%$ for positive and negative results, respectively, in the test set. Similarly, it moved from $12 \%$ to $72 \%$ and to $4 \%$ for positive and negative results, respectively, in the validation set (Data Supplement).

\section{Improves Categorization and Prognosis of PTCL}

We then tested the potential nosologic and clinical impacts of the MC. To do this, we first applied the model to a panel of six PTCLs NOS

\begin{tabular}{|c|c|c|}
\hline \multirow[b]{2}{*}{ GEP } & \multicolumn{2}{|c|}{ Histopathology } \\
\hline & ALK-Negative ALCL & PTCL NOS \\
\hline ALK-negative ALCL & 6 & 1 \\
\hline \multirow[t]{2}{*}{ PTCL NOS } & 0 & 54 \\
\hline & \multicolumn{2}{|c|}{ Histopathology } \\
\hline Accuracy & Value (\%) & $95 \% \mathrm{Cl}$ \\
\hline ST & 100 & 100 to 100 \\
\hline SP & 98 & 95 to 100 \\
\hline PPV & 86 & 60 to 100 \\
\hline NPV & 100 & 100 to 100 \\
\hline \multicolumn{3}{|l|}{ LR } \\
\hline Positive & 55 & 7.89 to 383.54 \\
\hline Negative & NC & NC \\
\hline Overall accuracy & $98^{*}$ & \\
\hline Pretest probability & 10 & 2 to 17 \\
\hline \multicolumn{3}{|c|}{$\begin{array}{l}\text { Abbreviations: ALCL, anaplastic large-cell lymphoma; GEP, gene expression } \\
\text { profile; LR, likelihood ratio; NC, not calculable (tends to zero); NOS, not } \\
\text { otherwise specified; NPV, negative predictive value; PPV, positive predictive } \\
\text { value; PTCL, peripheral T-cell lymphoma; SP, specificity; ST, sensitivity. } \\
{ }^{*} 60 \text { of } 61 \text {. }\end{array}$} \\
\hline
\end{tabular}

\begin{tabular}{lcc}
\multicolumn{3}{c}{ Table 3. Diagnostic Accuracy of AlTL Molecular Classifier Evaluated in } \\
Validation Set
\end{tabular}

with T-follicular helper (Tfh) phenotype but lacking a morphology consistent with either AITL or PTCL NOS follicular variant. Notably, all six patient cases were predicted to be PTCL NOS, providing further evidence that a subset of PTCL NOS shares a Tfh derivation but is distinct from AITL.

Furthermore, we evaluated the impact of the MC in PTCL prognostication. We included 203 of 244 patient cases for which complete information was available; median follow-up of living patients was 1,088 days (range, 14 to 4,664 days). The median OS for the entire

Table 4. Diagnostic Accuracy of ALK-Negative ALCL Molecular Classifier Evaluated in Validation Set

\begin{tabular}{|c|c|c|}
\hline \multirow[b]{2}{*}{ GEP } & \multicolumn{2}{|c|}{ Histopathology } \\
\hline & ALK-Negative ALCL & PTCL NOS \\
\hline ALK-negative ALCL & 8 & 3 \\
\hline \multirow[t]{2}{*}{ PTCL NOS } & 3 & 75 \\
\hline & \multicolumn{2}{|c|}{ Histopathology } \\
\hline Accuracy & Value (\%) & $95 \% \mathrm{Cl}$ \\
\hline ST & 72.73 & 46 to 99 \\
\hline SP & 96.15 & 92 to 100 \\
\hline PPV & 72.73 & 46 to 99 \\
\hline NPV & 96.15 & 92 to 100 \\
\hline \multicolumn{3}{|l|}{ LR } \\
\hline Positive & 18.91 & 5.89 to 60.75 \\
\hline Negative & 0.28 & 0.11 to 0.75 \\
\hline Overall accuracy & $93^{*}$ & \\
\hline Pretest probability & 12.36 & 6 to 19 \\
\hline \multicolumn{3}{|c|}{$\begin{array}{l}\text { Abbreviations: ALCL, anaplastic large-cell lymphoma; GEP, gene expression } \\
\text { profile; LR, likelihood ratio; NC, not calculable (tends to zero); NOS, not } \\
\text { otherwise specified; NPV, negative predictive value; PPV, positive predictive } \\
\text { value; PTCL, peripheral T-cell lymphoma; SP, specificity; ST, sensitivity. } \\
\text { *83 of } 89 \text {. }\end{array}$} \\
\hline
\end{tabular}


population was 469 days; 3-year OS rates were 16\%, 44\%, and 19\% for patients with AITL, ALK-negative ALCL, and PTCL NOS, respectively. We then calculated the OS curves for patient cases classified according to either conventional histopathology or GEP. Remarkably, although with conventional methods we only observed a trend in favor of ALK-negative ALCL versus PTCL NOS (median OS, 1,484 v 395 days; $P=.62$; Fig 2 A), when patient cases were reclassified by GEP, the survival difference between these two groups became statistically significant (median OS, 1,570 v 391 days; $P=.011$; Fig 2B). On the
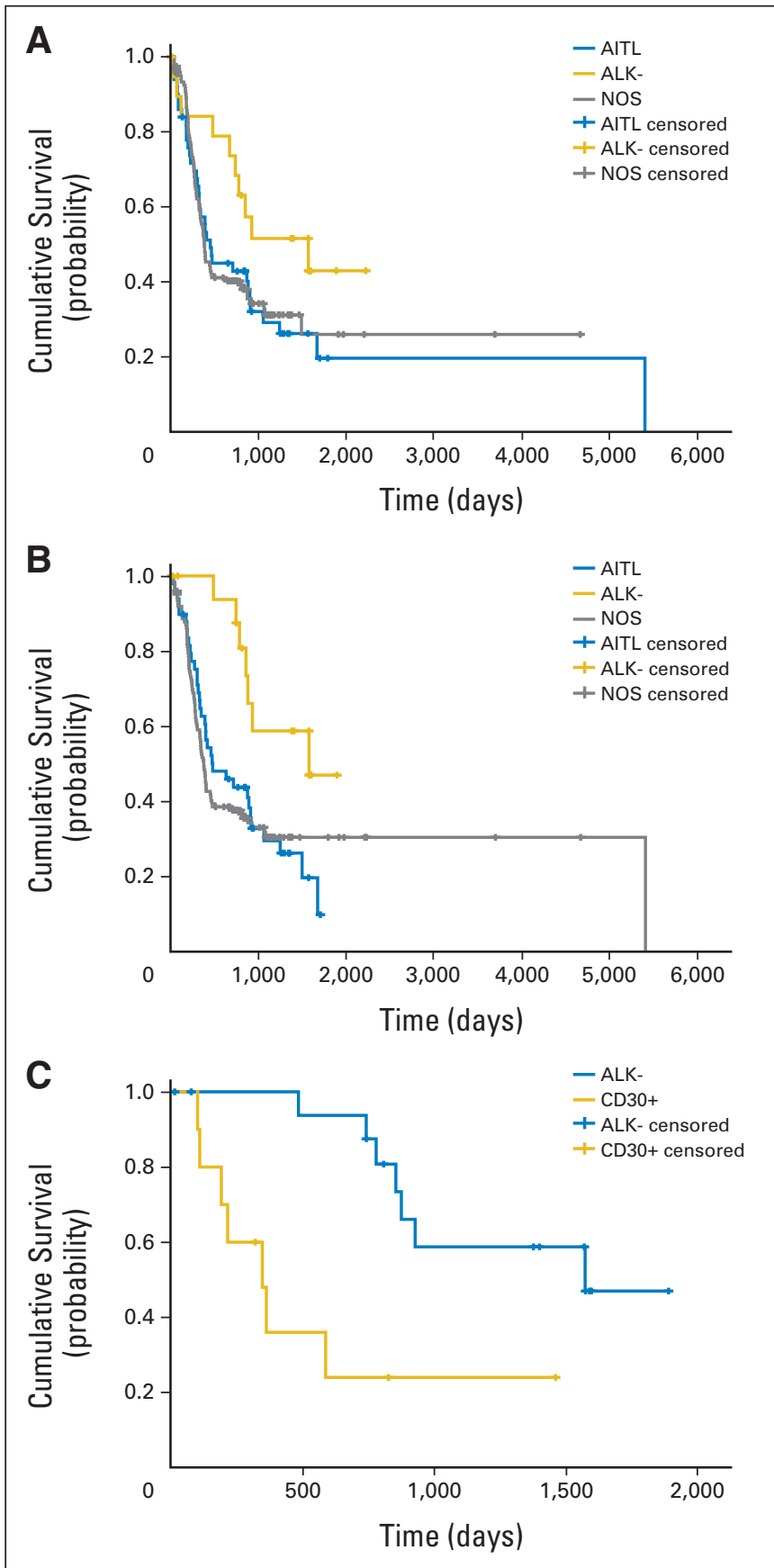

Fig 2. Survival curves according to peripheral T-cell lymphoma (PTCL): (A) histopathologic subtype, (B) molecular subtype, and (C) molecular distinction of CD30+ PTCL not otherwise specified (NOS) and ALK-negative anaplastic largecell lymphoma (ALK-). AITL, angioimmunoblastic T-cell lymphoma. contrary, no differences in survival were noted when PTCL NOS was compared with AITL. Moreover, we tested 14 patient cases of PTCL with strong CD30 expression but lack of typical ALCL morphology, for which a consensus histopathologic diagnosis could not be reached between PTCL NOS and ALK-negative ALCL. All patient cases were classified as PTCL NOS (Data Supplement). Most importantly, the OS of these patients (10 of 14 were evaluable) turned out to be significantly worse than that of patients with ALK-negative ALCL (median OS, $1,570 v 333$ days; $P=.02$; Fig 2C), indicating the usefulness of the tool in this peculiar setting as well. Details are provided in the Data Supplement. Together, these data demonstrate the clinical utility of the proposed tools.

\section{DISCUSSION}

In this article, we describe the use of GEP for the differential diagnosis of the commonest nodal PTCLs by focusing on the distinction of either AITL or ALK-negative ALCL from PTCL NOS, which may be of remarkable clinical interest and not always clear cut based on WHO classification criteria. ${ }^{18}$ Specifically, the morphologic features of AITL (clear-cell cytology, follicular dendritic cell meshwork, and abundant arborized high endothelium venules) are matters of subjective evaluation and can be partly observed in some PTCLs NOS. ${ }^{20,21}$ Furthermore, a proportion of PTCLs NOS present with the typical phenotype of AITL cells, reflecting their correspondence to normal Tfh lymphocytes (CD4+, CXCL13+, ICOS +, PD1 +, CD10+ and CD10-, and BCL6+ and BCL6- )., ${ }^{9,20,22,23}$ In this regard, we offer objective evidence that such tumors, although sharing the cellular counterpart, do not correspond to AITL. This finding suggests that a novel category of Tfh-related PTCLs might be recognized in the future. Importantly, in the last few months, somatic mutations of IDH2 and TET2 were found to be associated with AITL, the latter also observed in Tfh PTCL NOS. ${ }^{24,25}$ Additionally, Feldman et $\mathrm{al}^{26}$ identified $\mathrm{t}(6 ; 7)(\mathrm{p} 25.3 ; \mathrm{q} 32.3)$ as a specific lesion of ALCL. Therefore, although occurring only in a fraction of patient cases, their detection can certainly strengthen diagnosis of the different PTCL subtypes. Furthermore, PTCLs may present with numerous large cells and CD30 expression, ${ }^{20}$ making the differential diagnosis between PTCL NOS and ALK-negative ALCL problematic. In our series, GEP was able to discriminate AITL and ALK-negative ALCL from PTCL NOS with remarkable efficiency, with overall diagnostic accuracy varying from $77 \%$ to $98 \%$. Notably, PPV and NPV were also significant, with each test resulting in at least $86 \%$ for one of the values. Of note, when we evaluated the ability of the MCs to make a difference between pre- and post-test patient probabilities of having the given disease (ie, real potential for clinical application), we found that they were highly effective (Data Supplement). Particularly, both the classifiers, having high SP (80\% to 100\%), turned out to be good as confirmatory tests or SPIN (Specificity Rule In) tests (ie, if positive, they confirm presence of condition). Similarly, with ST values ranging from $72 \%$ to $100 \%$, they also performed well as screening tests or SNOUT (Sensitivity Rule Out) tests (ie, if negative, they rule out disease).

Indeed, this phase III diagnostic accuracy study represents, to our knowledge, a unique example available in this field. Previously, a few studies in the last years dealt with GEP of PTCL, providing the basic evidence that different entities could be distinguished based on their specific GEP. In particular, Martinez-Delgado et $\mathrm{al}^{27}$ showed that 
PTCL differed from T-lymphoblastic lymphoma. Subsequently, Ballester et $\mathrm{al}^{28}$ indicated that AITL and ALCL could be separate from the other subtypes, although PTCLs/NOS still remained mixed up. Finally, Piccaluga et $a l,{ }^{7,8}$ de Leval et al, ${ }^{9}$ Iqbal et $\mathrm{al}^{10}{ }^{10}$ and Piva et $\mathrm{al}^{11}$ showed that PTCL NOS, AITL, and ALK-negative ALCL could be discriminated based on their GEP. ${ }^{7-11}$ Together, these studies demonstrated that the different PTCL subtypes retained molecular differences that might be used for their differential diagnosis. However, they did not test potential diagnostic accuracy. In addition, prognostic impact was limited to the distinction of cytotoxic versus helper PTCL $\mathrm{NOS}^{7-10}$ and to the description of a proliferation signature associated with worse outcome, this being in line with immunohistochemical evidence. ${ }^{29,30}$

Conversely, we developed an MC able to improve the current stratification of patients with PTCL based on conventional histopathology with remarkable prognostic impact. In fact, using the MC, we could distinguish patient cases with significantly different clinical outcomes. Additionally, we had the opportunity to study a series CD30+ PTCL patient cases, the diagnosis of which was matter of debate among experienced hematopathologists. Strikingly, the classifier identified all of them as PTCLs NOS, not ALCLs. This distinction was not trivial. In fact, using GEP, we could discriminate a group of CD30+ PTCLs NOS with significantly worse outcomes than ALK-negative ALCLs. Importantly, the inclusion of CD30+ forms within PTCL NOS rather than within ALCL is still largely discussed in the scientific community. Here, we provide for the first time to our knowledge objective evidence that CD30+ PTCL NOS is distinct from ALKnegative ALCL, with the two diseases characterized by significantly different prognoses.

Notably, on the basis of gene ontology enrichment analysis, we did not have evidence supporting the role of tumor content in the signature generation. Of course, we cannot exclude a minimal impact on single-gene expression. However, the validation of the signatures across three different data sets makes any significant effect of aspecific components extremely unlikely. Furthermore, we could rule out a possible puzzling effect of the microenvironment on class prediction in all patient cases for which diagnostic material was still available. It should be underlined that the tool was designed for the routine diagnostic use, and the FFPE samples included abundant reactive cells that were correctly classified.

Interestingly, in this study, we applied a novel microarray technology able to generate GEP from FFPE routine tissues. ${ }^{31}$ Of note, we obtained a consistent profile in all patient cases examined. Indeed, this is clinically relevant. In fact, GEP has failed so far to become an integral part of diagnostic hematopathology, remaining limited to the field of research, largely because of the need for fresh/frozen material, often not available in the routine setting. Certainly, DASL whole-genome GEP can be reliably performed on both fresh and FFPE tissues, using low amounts of RNA. ${ }^{31}$ Of note, we were able to confirm the consistency of this tool in a large panel of B- and T-cell lymphomas by matching gene and protein expression (data not shown), as other groups have done. ${ }^{32}$ In addition, we showed that the molecular signatures identified in this study could be successfully applied in an independent validation set of patients cases for which GEP was generated from frozen tissues with different platforms (Illumina, San Diego, CA $v$ Affymetrix, Santa Clara, CA). On the other hand, signatures generated on fresh/frozen samples were successfully applied to our series (Data Supplement).

Although the study design (phase III) does not allow an index test to overcome the reference standard by definition, the proposed classifiers performed well. Our findings support the use of an MC as an additional tool in the diagnostic workup of nodal PTCLs. In fact, the aim of our study was to provide pathologists with another robust diagnostic tool integrating morphology and phenotype. Bearing this in mind, it is noteworthy that we were able to significantly limit the number of genes used for an efficient classification, thus making it possible to quickly define a custom assay specific to this issue, efficient in FFPE samples. In conclusion, our study identified gene signatures able to efficiently differentiate nodal PTCLs, starting from FFPE tissues, which may become useful tools in the diagnostic workup of these diseases.

Bologna University obtained a patent from the Italian Patent and Trademark Office for the molecular signatures used for the diagnostic classifier (patent No. 61.U2164.12.IT.34).

\section{AUTHORS' DISCLOSURES OF POTENTIAL CONFLICTS OF INTEREST}

The author(s) indicated no potential conflicts of interest.

\section{AUTHOR CONTRIBUTIONS}

Provision of study materials or patients: Sylvia Hartmann, Martin L. Hansmann, Roberto Piva, Javeed Iqbal, John C. Chan, Dennis Weisenburger, Julie M. Vose, Massimo Federico, Giorgio Inghirami, Pier Luigi Zinzani, Stefano A. Pileri

Collection and assembly of data: Clara Bertuzzi, Maura Rossi, Francesco Bacci, Elena Sabattini, Claudio Agostinelli, Anna Gazzola, Maria

Antonella Laginestra, Claudia Mannu, Maria Rosaria Sapienza, Sylvia Hartmann, Martin L. Hansmann, Roberto Piva, Javeed Iqbal, John C. Chan, Dennis Weisenburger, Julie M.Vose, Monica Bellei, Massimo Federico, Giorgio Inghirami, Pier Luigi Zinzani, Stefano A. Pileri Data analysis and interpretation: Pier Paolo Piccaluga, Fabio Fuligni, Antonio De Leo, Clara Bertuzzi

Manuscript writing: All authors

Final approval of manuscript: All authors

\section{REFERENCES}

1. Jaffe ES, Harris NL, Stein $H$, et al: Introduction and overview of the classification of the lymphoid neoplasms, in Swerdlow S, Campo E, Harris NL, et al (eds): WHO Classification of Tumours of Hematopoietic and Lymphoid Tissues (ed IV). Lyon, France, IARC Press, 2008, pp 158-166

2. Federico M, Bellei M, Pesce $E$, et al: T-cell project: An international, prospective, observational study of patients with aggressive peripheral T-cell lymphoma-Analysis of first 524 patients. Presented at the 11th International Conference on Malignant Lymphoma, Lugano, Switzerland, June 15-18, 2011

3. Savage KJ, Harris NL, Vose JM, et al: ALKanaplastic large-cell lymphoma is clinically and immunophenotypically different from both ALK+ ALCL and peripheral T-cell lymphoma, not otherwise specified: Report from the International Peripheral T-Cell Lymphoma Project. Blood 111: 5496-5504, 2008
4. Roncolato F, Gazzola A, Zinzani PL, et al: Targeted molecular therapy in peripheral T-cell lymphomas. Expert Rev Hematol 4:551-562, 2011

5. Hummel M, Bentink S, Berger $H$, et al: A biologic definition of Burkitt's lymphoma from transcriptional and genomic profiling. N Engl J Med 354:2419-2430, 2006

6. Dave SS, Fu K, Wright GW, et al: Molecular diagnosis of Burkitt's lymphoma. N Engl J Med 354:2431-2442, 2006 
7. Piccaluga PP, Agostinelli C, Califano A, et al: Gene expression analysis of peripheral T cell lymphoma, unspecified, reveals distinct profiles and new potential therapeutic targets. J Clin Invest 117:823-834, 2007

8. Piccaluga PP, Agostinelli C, Califano A, et al: Gene expression analysis of angioimmunoblastic lymphoma indicates derivation from $T$ follicular helper cells and vascular endothelial growth factor deregulation. Cancer Res 67:10703-10710, 2007

9. de Leval L, Rickman DS, Thielen $C$, et al: The gene expression profile of nodal peripheral T-cell lymphoma demonstrates a molecular link between angioimmunoblastic T-cell lymphoma (AITL) and follicular helper T (TFH) cells. Blood 109:4952-4963, 2007

10. Iqbal J, Weisenburger DD, Greiner TC, et al: Molecular signatures to improve diagnosis in peripheral T-cell lymphoma and prognostication in angioimmunoblastic T-cell lymphoma. Blood 115: 1026-1036, 2010

11. Piva R, Agnelli L, Pellegrino E, et al: Gene expression profiling uncovers molecular classifiers for the recognition of anaplastic large-cell lymphoma within peripheral T-cell neoplasms. J Clin Oncol 28:1583-1590, 2010

12. Piccaluga $\mathrm{PP}$, Agostinelli $C$, Zinzani $\mathrm{PL}$, et al: Expression of platelet-derived growth factor receptor alpha in peripheral T-cell lymphoma not otherwise specified. Lancet Oncol 6:440, 2005

13. Piccaluga PP, De Falco G, Kustagi $M$, et al: Gene expression analysis uncovers similarity and differences among Burkitt lymphoma subtypes. Blood 117:3596-3608, 2011

14. National Center for Biotechnology Information: Gene expression omnibus. http://www.ncbi.nlm.nih.gov/ projects/geo
15. Standards for the Reporting of Diagnostic Accuracy Studies: STARD statement. http://www.stardstatement.org

16. McShane LM, Altman DG, Sauerbrei $W$, et al: Reporting recommendations for tumor marker prognostic studies. J Clin Oncol 23:9067-9072, 2005

17. Whiting PF, Rutjes AW, Westwood ME, et al: QUADAS-2: A revised tool for the quality assessment of diagnostic accuracy studies. Ann Intern Med 155:529-536, 2011

18. Swerdlow S, Campo E, Harris NL, et al (eds): WHO Classification of Tumours of Hematopoietic and Lymphoid Tissues (ed IV). Lyon, France, IARC Press, 2008

19. Kaplan E, Meier P: Non-parametric estimation from incomplete observation. JAMA 58:457-481, 1958

20. Pileri SA, Weisenburger DD, Sng I, et al: Peripheral T-cell lymphoma, not otherwise specified, in Swerdlow S, Campo E, Harris NL, et al (eds): WHO Classification of Tumours of Hematopoietic and Lymphoid Tissues (ed IV). Lyon, France, IARC Press, 2008, pp 306-308

21. Dogan A, Gaulard P, Jaffe ES, et al: Angioimmunoblastic T-cell lymphoma, in Swerdlow $\mathrm{S}$, Campo E, Harris NL, et al (eds): WHO Classification of Tumours of Hematopoietic and Lymphoid Tissues (ed IV). Lyon, France, IARC Press, 2008, pp 309-311

22. Agostinelli C, Hartmann S, Klapper W, et al: Peripheral $T$ cell lymphomas with follicular $T$ helper phenotype: A new basket or a distinct entity? Revising Karl Lennert's personal archive. Histopathology 59:679-691, 2011

23. Piccaluga PP, Agostinelli $C$, Tripodo $C$, et al: Peripheral T-cell lymphoma classification: The matter of cellular derivation. Expert Rev Hematol 4:415425, 2011
24. Cairns RA, labal J, Lemonnier $F$, et al: IDH2 mutations are frequent in angioimmunoblastic T-cell lymphoma. Blood 119:1901-1903, 2012

25. Lemonnier F, Couronné L, Parrens $M$, et al: Recurrent TET2 mutations in peripheral T-cell lymphomas correlate with TFH-like features and adverse clinical parameters. Blood 120:1466-1469, 2012

26. Feldman $A L$, Dogan $A$, Smith $D I$, et al: Discovery of recurrent $t(6 ; 7)(p 25.3 ; q 32.3)$ translocations in ALK-negative anaplastic large cell lymphomas by massively parallel genomic sequencing. Blood 117: 915-919, 2011

27. Martinez-Delgado $B$, Meléndez $B$, Cuadros $M$, et al: Expression profiling of T-cell lymphomas differentiates peripheral and lymphoblastic lymphomas and defines survival related genes. Clin Cancer Res 10:4971-4982, 2004

28. Ballester B, Ramuz $O$, Gisselbrecht $C$, et al: Gene expression profiling identifies molecular subgroups among nodal peripheral T-cell lymphomas. Oncogene 25:1560-1570, 2006

29. Cuadros M, Dave SS, Jaffe ES, et al: Identification of a proliferation signature related to survival in nodal peripheral T-cell lymphomas. J Clin Oncol 25:3321-3329, 2007

30. Went P, Agostinelli C, Gallamini A, et al: Marker expression in peripheral T-cell lymphoma: $A$ proposed clinical-pathologic prognostic score. J Clin Oncol 24:2472-2479, 2006

31. April C, Klotzle B, Royce $T$, et al: Wholegenome gene expression profiling of formalin-fixed, paraffin-embedded tissue samples. PLoS One 4:e8162, 2009

32. Waddell N, Cocciardi S, Johnson J, et al: Gene expression profiling of formalin-fixed, paraffinembedded familial breast tumours using the whole genome-DASL assay. J Pathol 221:452-461, 2010

\section{Affiliations}

Pier Paolo Piccaluga, Fabio Fuligni, Antonio De Leo, Clara Bertuzzi, Maura Rossi, Francesco Bacci, Elena Sabattini, Claudio Agostinelli, Anna Gazzola, Maria Antonella Laginestra, Claudia Mannu, Maria Rosaria Sapienza, Pier Luigi Zinzani, and Stefano A. Pileri, S. Orsola-Malpighi Hospital, University of Bologna, Bologna; Roberto Piva and Giorgio Inghirami, University of Torino, Torino; Monica Bellei and Massimo Federico, Università di Modena e Reggio Emilia, Modena, Italy; Sylvia Hartmann and Martin L. Hansmann, Goethe University, Frankfurt am Main, Germany; Javeed Iqbal, John C. Chan, and Julie M. Vose, University of Nebraska Medical Center, Omaha, NE; Denis Weisenburger, City of Hope National Medical Center, Duarte, CA. 


\section{Acknowledgment}

We thank the many collaborators at the participating centers. 